\title{
Wavelet phase coherence analysis: Application to skin temperature and blood flow
}

\author{
Andriy Bandrivskyy ", Alan Bernjak*, Peter McClintock and Aneta \\ Stefanovska* \\ "Department of Physics, Lancaster University, Lancaster LA1 $4 Y B$, UK \\ * Group of Nonlinear Dynamics and Synergetics, Faculty of Electrical Engineering, \\ University of Ljubljana, Tržaška 25, 1000 Ljubljana, Slovenia
}

\begin{abstract}
The technique of wavelet phase coherence analysis is introduced and used to explore relationships between oscillations on blood flow and temperature in the skin of 10 healthy subjects. Their skin temperature and blood flow were continuously recorded: under basal conditions for 30 minutes; during local cooling of the skin with an ice-pack for 20 minutes: and 30 minutes thereafter. The group mean basal skin temperature of $33.4^{\circ} \mathrm{C}$ was decreased to $29.2^{\circ} \mathrm{C}$ during the cooling period, and had recovered to $32.1^{\circ} \mathrm{C}$ by the end of the recording. The wavelet transform was used to obtain the time-frequency content of the two signals, and their coherence. It is shown that cooling increases coherence to a statistically significant extent in two frequency intervals, around 0.007 and $0.1 \mathrm{~Hz}$, suggesting that these oscillatory components are involved in the regulation of skin temperature when cold is applied as a stress.
\end{abstract}

Keywords: Blood flow, skin temperature regulation, oscillations, time series analysis, wavelets, phase coherence

\section{Introduction}

Analysis of time series is crucial to the understanding of complex systems and how they work. The existence of complexity implies that several different processes are present in the system's dynamics. Often, time series of two or more different variables can be measured. An appropriately chosen form of time series analysis may be able to establish the interrelationships, if any, between the time series and thus yield information relevant to the underlying processes that generate them.

The analysis of multivariate data of this kind has recently attracted much attention, including applications such as synchronization analysis (Pikovsky et al., 2001) and the detection of coupling direction (Rosenblum et al., 2002; Paluš and Stefanovska, 2003). The methods suggested by these authors require, however, that distinct oscillatory modes are present and that they can be unambiguously distinguished in the time series under study.

Unfortunately this is not always so. For example, although it was suggested that the dynamics of cardiovascular signals can be described 
as a few oscillatory modes (Stefanovska et al., 2001), not all the modes can be measured independently. In addition, partly on account of the finite time of observation, the low frequencies modes cannot easily be decomposed or separated from the noisy background. Additional complications are that signals from complex systems often involve modes at several frequencies and that the latter may themselves be time-variable.

The cross-spectrum can be used to detect whether two processes involve oscillations within the same frequency region. However, the limitation of this technique lies in its inability to distinguish whether the oscillations at a given frequency are independent or mutually related. A different approach is therefore required, able to detect phase coherence in different frequency regions of two given signals. We use the term phase coherence here in the generalised sense that a phase difference between two oscillations is allowed provided that it remains constant throughout the whole time of observation.

In what follows, we apply a wavelet-based technique to detect phase coherence, enabling us to introduce a technique for studying interactions between skin blood flow and temperature regulatory mechanisms (Johnson et al., 1986; Shusterman et al., 1997; Nuzzaci et al., 1999).

\section{Methods}

\subsection{Measurements}

Simultaneous measurements were made of skin temperature and blood flow for 10 healthy subjects, 7 male and 3 female, aged between 24 and 62 years (median age 30), as follows. The subject relaxed supine on a bed for 15 minutes. A temperature sensor (Thermilinear ${ }^{\circledR}$, YSI Incorporated, Ohio, USA) connected to a signal conditioning unit (Cardiosignals, Jožef Stefan Institute, Slovenia) and a laser-Doppler blood flow sensor (DP1T-V2 probe connected to DRT4, Moor Instruments, Axminster, Devon, UK) were placed together on the volar aspect of the forearm, positioned so as to avoid large blood vessels or blemishes, held in position with a double-sided adhesive annulus.

For a continuous period of 80 minutes, temperature and blood flow data were digitized (16-bit at a sampling frequency of $400 \mathrm{~Hz}$ ) and recorded. After 30 minutes of basal recording, a thermal shock was used to enhance oscillatory activity at the characteristic frequencies associated with temperature regulation: an ice-pack was applied to the measurement area and its immediate vicinity, through a layer of fabric to lessen the thermal shock. After 20 minutes, the ice-pack was removed, while the recording was continued for another 30 minutes. 


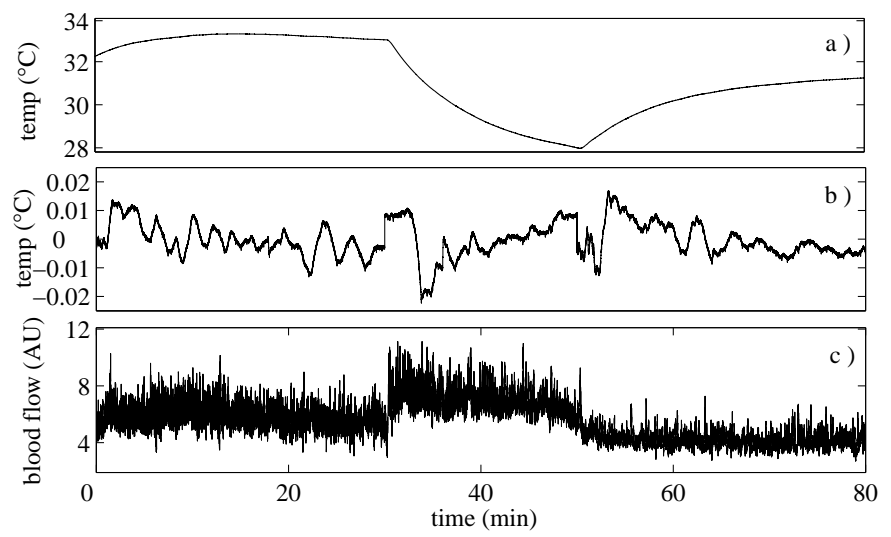

Figure 1. Temperature (a), its detrended value (b) and the skin blood flow (c) recorded on the volar side of the left arm as described in Sec. 2.1.

The resolution of temperature $\theta$ was $0.0003{ }^{\circ} \mathrm{C}$ within $20<\theta<40^{\circ} \mathrm{C}$ using 16-bit bipolar A/D conversion scaled to $\pm 5 \mathrm{~V}$. A typical set of data, measured in this way for one subject, is shown in Fig. 1.

\subsection{Generalized Phase COHEREnCE}

To provide for time-frequency analysis of these bivariate data, we have developed a technique that makes use of the wavelet transform. It is based on the idea that the way of the splitting of the time interval $T$ (the whole length of time series) should be different for different basic frequencies $\omega_{k}$, and that the scaling of $\omega_{k}$ should be logarithmic. The continuous wavelet transform is defined as

$$
X(\omega, t)=\int_{-\infty}^{\infty} \Psi_{\omega, t}(u) x(u) d u,
$$

where $\Psi_{\omega, t}$ is a family of generally nonorthogonal basis functions. These are obtained by scaling and shifting the basic window function $\psi(u)$ in the time domain

$$
\Psi_{\omega, t}=\frac{\sqrt{\omega}}{\sqrt{2 \pi}} \psi\left(\frac{n-t}{2 \pi} \omega\right)
$$

Different window functions can be used. We have chosen the Morlet wavelet. Its simplified expression in the time domain is

$$
\psi(u)=\frac{1}{\sqrt[4]{\pi}} e^{-i 2 \pi u} e^{-u^{2} / 2} .
$$


In numerical applications, frequency and time are restricted to discrete values only. An approximation of the continuous wavelet transform was calculated using a discrete wavelet family. The basic frequency is scaled according to $\omega_{k}=\omega_{k-1} 1.05$ and the time is discretised by $t_{n}=n \tau ; \tau=1 \mathrm{~s}$. The choice of the length of the window is a tradeoff between good and bad resolution in frequency and time domains. In our case the length of the window was determined by the amplitude of the window. The boundary value of the window was reached, when it's amplitude was smaller than 0.00001. For a discussion of wavelet analysis, its application to cardiovascular signals, and optimal choice of parameters, see the review by Bračič and Stefanovska (1998).

The complex spectral function $X\left(\omega_{k}, t_{n}\right)=X_{k, n}=a_{k, n}+i b_{k, n}$ describes the spectral properties of the signal $x(t)$ in the time-frequency domain. For each time $t_{n}$ and frequency $\omega_{k}$ amplitude $\left|X_{k, n}\right|=\left(a_{k, n}^{2}+b_{k, n}^{2}\right)^{1 / 2}$ and phase $\phi_{k, n}=\arctan \left(b_{k, n} / a_{k, n}\right)$ are evaluated. For two signals $x_{1}(t)$ and $x_{2}(t)$ one gets two phases $\phi_{1 k, n}$ and $\phi_{2 k, n}$ and the relative phase difference $\Delta \phi_{k, n}=\phi_{2 k, n}-\phi_{1 k, n}$ can be computed. Then

$$
\begin{aligned}
\cos \Delta \phi_{k, n} & =\frac{a_{1 k, n} a_{2 k, n}+b_{1 k, n} b_{2 k, n}}{\sqrt{a_{1 k, n}^{2}+b_{1 k, n}^{2}} \sqrt{a_{2 k, n}^{2}+b_{2 k, n}^{2}}}, \\
\sin \Delta \phi_{k, n} & =\frac{b_{1 k, n} a_{2 k, n}-a_{1 k, n} b_{2 k, n}}{\sqrt{a_{1 k, n}^{2}+b_{1 k, n}^{2}} \sqrt{a_{2 k, n}^{2}+b_{2 k, n}^{2}}} .
\end{aligned}
$$

Coefficients $\cos \Delta \phi_{k, n}$ and $\sin \Delta \phi_{k, n}$ can be averaged in time for the whole length of the time series

$$
\begin{aligned}
\left\langle\cos \Delta \phi_{k, n}\right\rangle & =\frac{1}{M_{k}} \sum_{n=1}^{M_{k}} \cos \Delta \phi_{k, n}, \\
\left\langle\sin \Delta \phi_{k, n}\right\rangle & =\frac{1}{M_{k}} \sum_{n=1}^{M_{k}} \sin \Delta \phi_{k, n},
\end{aligned}
$$

and the time-averaged wavelet phase coherence function is then defined as

$$
C_{\phi}\left(\omega_{k}\right)=\sqrt{\left\langle\cos \Delta \phi_{k, n}\right\rangle^{2}+\left\langle\sin \Delta \phi_{k, n}\right\rangle^{2}} .
$$

The function $C_{\phi}\left(\omega_{k}\right)$ takes values between 0 and 1 . If $C_{\phi}\left(\omega_{k}\right) \simeq 0$, then there is no phase coherence between the signals $x_{1}(t)$ and $x_{2}(t)$ at the frequency $\omega_{k}$. The case when $C_{\phi}\left(\omega_{k}\right)>0$ is less well defined. The meaning of a nonzero phase coherence function for any given frequency $\omega_{k}$ depends crucially on the length $T$ of the whole time series. It is $T$ 


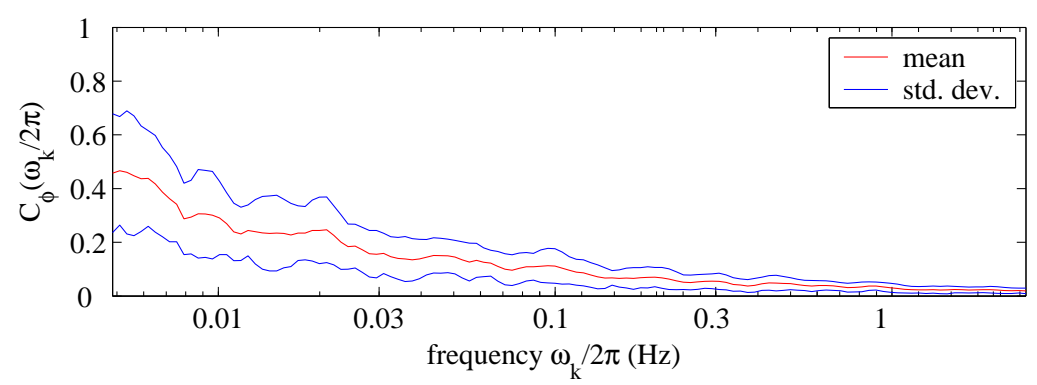

Figure 2. The mean (red line) and standard deviation (blue lines) of $C_{\phi}\left(\omega_{k}\right)$ for two uncorrelated white Gaussian noise signals of finite length.

that limits our ability to average in time the coefficients $\cos \Delta \phi_{k, n}$ and $\sin \Delta \phi_{k, n}$. For example, in the extreme case when $C_{\phi}\left(\omega_{k}\right)$ is evaluated for the lowest possible frequency $\omega_{1}=2 \pi q / T_{1}=2 \pi q / T$ one gets a trivial result $C_{\phi}\left(\omega_{1}\right)=1$. Thus the function $C_{\phi}\left(\omega_{k}\right)$ has an uninformative low frequency maximum, by construction. Therefore only the part of the coherence function for the higher frequencies $\left(\omega_{k} \gg \omega_{1}\right)$ can be used with confidence to evaluate the phase coherence between two signals. Within this range $C_{\phi}\left(\omega_{k}\right)=1$ would correspond to complete coherence, and $1>C_{\phi}\left(\omega_{k}\right)>0$ would correspond to partial coherence. The lower frequency region $\left(\omega_{k} \geq \omega_{1}\right)$ can be still used in a statistical sense, albeit with caution, when the properties of a set of signals are being compared.

An example of the uninformative low frequency peak is shown in Fig. 2 which shows the mean and standard deviation of $C_{\phi}\left(\omega_{k}\right)$ for two uncorrelated white Gaussian noise signals: 30 pairs of noise signals, each 30 minutes in length, were sampled at $10 \mathrm{~Hz}$, corresponding to the real data as measured.

\section{Skin temperature and blood flow interactions}

Fig. 3 shows the result of applying the wavelet transform to data like those of Fig. 1(b) and (c). The data were first resampled to $10 \mathrm{~Hz}$. Note that in the temperature very low frequency components dominate, while the blood flow signal contains several characteristic frequencies spanning the whole interval. While the frequency range of the cardiac pulse was unaffected, the oscillatory process around $0.1 \mathrm{~Hz}$, believed to originate from myogenic activity (Kvandal et al., 2003), decreased in frequency during cooling and gradually returned again towards its basal value after the ice was removed. Despite the poor time resolution 
a)
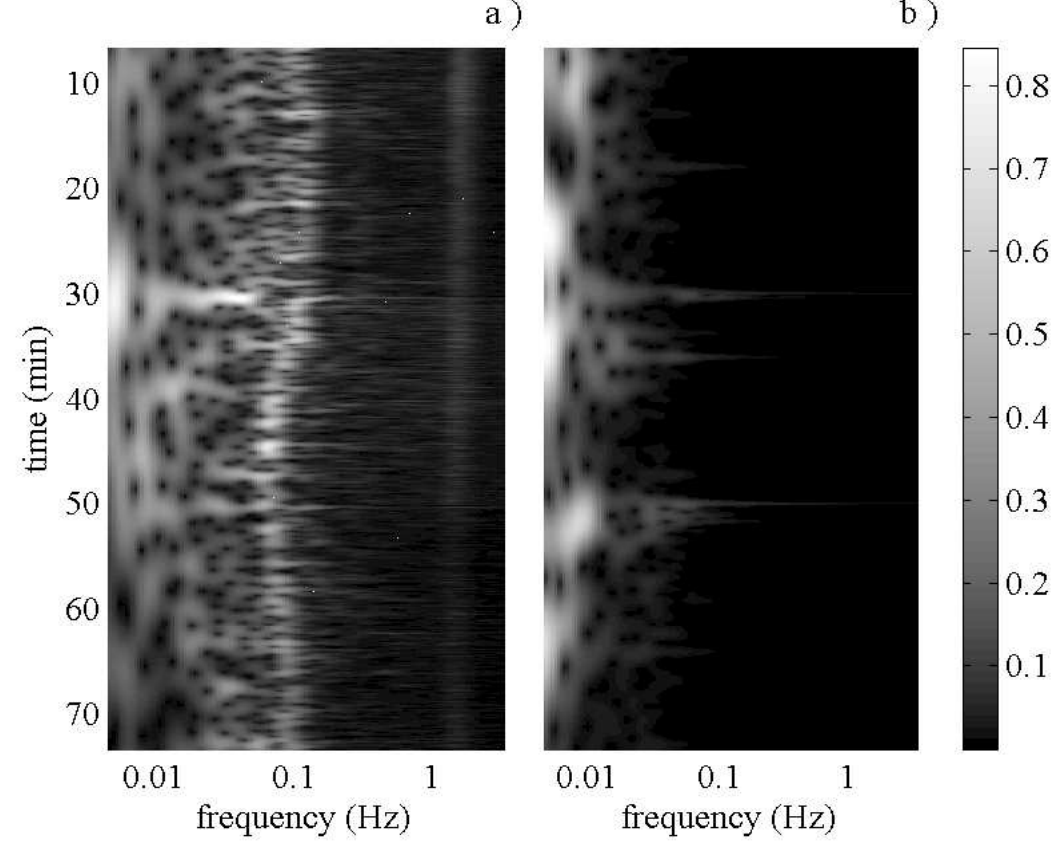

Figure 3. Time-frequency representation of (a) blood flow and (b) temperature signals obtained by wavelet transform.

in this low frequency range, there is clear evidence of a spectral peak at around $0.007 \mathrm{~Hz}$ that becomes intensified from about the time (30 minutes) when the ice-pack is applied. It is seen both in blood flow and in temperature. Note that the (lighter shaded) peaks near 30 and 50 minutes, affecting all frequencies, are artifacts due to the mechanical disturbance; the significant feature in (a) is the lighter shaded ridge at about $0.007 \mathrm{~Hz}$ running between $\sim 30$ and $\sim 54$ minutes.

The data have been subjected to statistical analyses to establish more quantitatively the response to cooling. Fig. 4 shows that the temperature decreases, and increases, significantly. Blood flow is slightly affected by the temperature stress (the time evolution obtained for one subject is shown in figure 1), but the differences are not statistically significant for the group as a whole. For the data labelled $\star \star \star, p=0$; and for that labelled $\star, p<0.01$.

An example of the calculated coherence function $C_{\phi}\left(\omega_{k}\right)$ is plotted in Fig. 5. It is generally higher than for uncorrelated white noise (Fig. 2), and there is some indication of increased coherence during cooling. To check this inference more carefully, the coherence was calculated for the group within different frequency intervals, yielding the results shown 

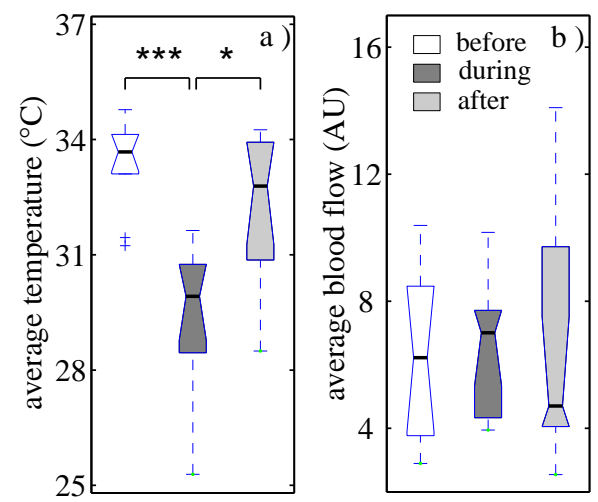

Figure 4. Group median, and boxplots of time averages of (a) skin temperature and (b) blood flow signals before, during and after cooling.

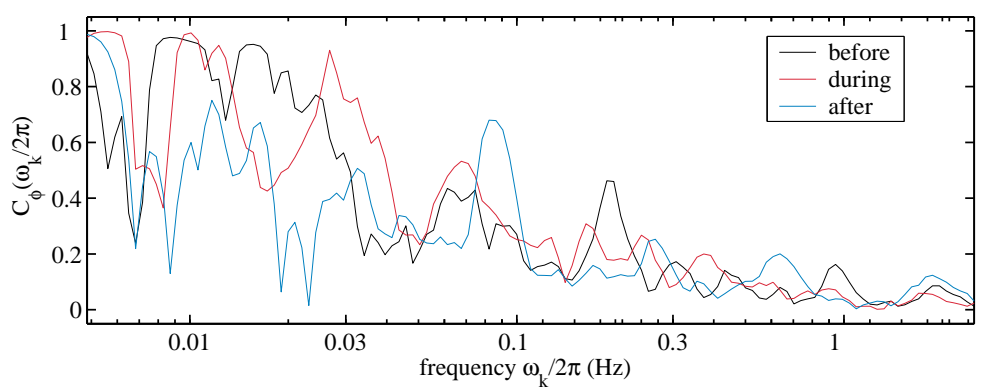

Figure 5. An example of the coherence between the skin temperature and blood flow oscillations as a function of frequency before, during and after cooling.

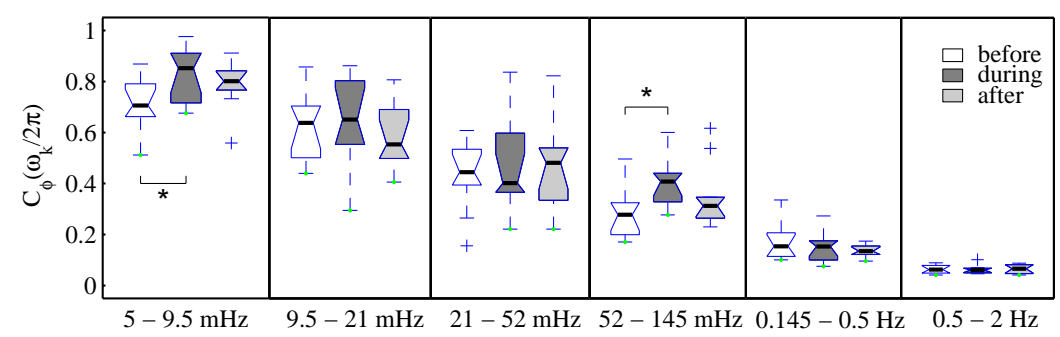

Figure 6. Group median, and boxplots of the coherence calculated within six frequency intervals (see text).

in Fig. 6. The five higher frequency intervals were those defined earlier (Bračič and Stefanovska, 1998), while the lowest frequency interval from 0.005 to $0.095 \mathrm{~Hz}$ has been included for the first time in this study. The coherence between temperature and blood flow oscillations increases to 
a statistically significant extent during cooling in two intervals: around $0.007 \mathrm{~Hz}$ and around $0.1 \mathrm{~Hz}$. While oscillations in the latter interval are hypothesized to originate from myogenic activity in the vessel walls (Kvandal et al., 2003), the slowest oscillations have not to our knowledge been discussed previously.

\section{Conclusion}

Wavelet-based coherence analysis has proved its worth by revealing the two frequency ranges that are apparently involved in temperature regulation. The existence of the higher of these, near $0.1 \mathrm{~Hz}$, is wellestablished. The lower range, around $0.007 \mathrm{~Hz}$, may perhaps relate to biochemical processes at the level of endothelium (Gustafsson et al., 1993; Kellogg et al., 2003; Kvandal et al., 2003) involved in the heat production that is increased in order to resist the reduction in temperature. Further work will be needed to establish whether this is in fact the case.

\section{Acknowledgements}

The work was supported by the Slovenian Ministry of Education, Science and Sport, the Engineering and Physical Sciences Research Council (UK), Leverhulme Trust, the British Council and INTAS.

\section{References}

Bračič, M. and A. Stefanovska: 1998, 'Wavelet based analysis of human blood flow dynamics'. Bull. Math. Biol. 60(5), 919-935.

Gustafsson, H., M. J. Mulvany, and H. Nilsson: 1993, 'Rhythmic contraction of isolated small arteries from rat - Influence of the endothelium'. Acta Physiol. Scand. 148(2), 153-163.

Johnson, J. M., G. Brengelmann, J. R. S. Hales, P. M. Vanhoutte, and C. B. Wenger: 1986, 'Regulation of the cutaneous circulation'. Federation Proceedings 45(13), 2841-2850.

Kellogg, D. L., J. L. Zhao, C. Friel, and L. J. Roman: 2003, 'Nitric oxide concentration increases in the cutaneous interstitial space during heat stress in humans'. J. Appl. Physiol. 94(5), 1971-1977.

Kvandal, P., A. Stefanovska, M. Veber, H. D. Kvernmo, and K.-A. Kirkebøen: 2003, 'Regulation of human cutaneous circulation evaluated by laser Doppler flowmetry, iontophoresis, and spectral analysis: importance of nitric oxide and prostaglandines'. Microvasc. Res. 65(3), 160-171.

Nuzzaci, G., A. Evangelisti, D. Righi, G. Giannico, and I. Nuzzaci: 1999, 'Is there any relationship between cold-induced vasodilatation and vasomotion?'. Microvasc. Res. 57(1), 1-7.

Paluš, M. and A. Stefanovska: 2003, 'Direction of coupling from phases of interacting oscillators: An information-theoretic approach'. Phys. Rev. E 67, 055201(R). 
Pikovsky, A., M. Rosenblum, and J. Kurths: 2001, Synchronization - A Universal Concept in Nonlinear Sciences. Cambridge: Cambridge University Press.

Rosenblum, M. G., L. Cimponeriu, A. Bezerianos, A. Patzak, and R. Mrowka: 2002, 'Identification of coupling direction: Application to cardiorespiratory interaction'. Phys. Rev. E. 65(4), 041909.

Shusterman, V., K. P. Anderson, and O. Barnea: 1997, 'Spontaneous skin temperature oscillations in normal human subjects'. Am. J. Physiol.: Regulatory Integrative Comp. Physiol. 273(3), R1173-R1181.

Stefanovska, A., M. Bračič Lotrič, S. Strle, and H. Haken: 2001, 'The cardiovascular system as coupled oscillators?'. Physiol. Meas. 22(3), 535-550. 
T_paper.tex; 16/04/2015; $16: 47 ;$ p.10 